\title{
Genital inoculation of male Macaca fascicularis with Neisseria gonorrhoeae and Ureaplasma urealyticum
}

\author{
WILLIAM R. BOWIE*, RONALD F. DIGIACOMO, KING K. HULMES AND \\ JAMES L. GALE
}

From the Department of Medicine, US Public Health Service Hospital; the Department of Epidemiology, School of Public Health and Community Medicine; and the Regional Primate Research Center, University of Washington, Seattle, Washington

SUMMARY Inoculation of the urethra, conjunctiva, pharynx, and anal canal of six male crab-eating macaques (Macaca fascicularis) with urethral exudate from male patients with urethral gonorrhoea or with laboratory gonococcal strains was unsuccessful in establishing infection or producing increased polymorphonuclear leucocytes on Gram stain. Intraurethral inoculation with laboratory strains of Ureaplasma urealyticum resulted in transiently positive urethral cultures for $U$. urealyticum and was associated in some cases with increased numbers of polymorphonuclear leucocytes on Gram stain of urethral material. The findings suggest that the crab-eating macaque may be useful for studying the pathogenesis of urethral infection with $U$. urealyticum.

\section{Introduction}

Investigations of gonococcal and nongonococcal urethritis have been hampered by the lack of animal models in which urethral infection can be produced. Among nonhuman primates only chimpanzees (Pan troglodytes) have been convincingly infected with Neisseria gonorrhoeae (Lucas et al., 1971); other simians have proved resistant to urethral infection (DiGiacomo et al., 1977; Gale et al., 1977). Urethral infection with Chlamydia trachomatis has been produced in baboons (Papio cynocephalus) (DiGiacomo et al., 1975) and pigtailed macaques (Macaca nemestrina) but not in capuchins (Cebus albifrons) (Gale et al., 1977). Ureaplasma urealyticum urethral infection has been produced in one of two pig-tailed macaques but not in baboons or capuchins (Gale et al., 1977). Experiments with other simians may reveal a more suitable nonhuman primate model for studying the pathogenesis and immunity of urethral infection

*Currently at the University of British Columbia, Faculty of Medicine, Vancouver General Hospital, Vancouver, British Columbia, Canada, V5Z1M9

Address for reprints: W. R. Bowie, Faculty of Medicine, Vancouver General Hospital, Vancouver, British Columbia, Canada V5Z 1 M9

Received for publication 12 December 1977 with N. gonorrhoeae, C. trachomatis, and $U$. urealyticum. Furthermore, whereas $N$. gonorrhoeae and $C$. trachomatis are recognised causes of urethral disease, the role of $U$. urealyticum in urethral disease remains equivocal. Therefore, a previously untested simian species, the crab-eating macaque (Macaca fascicularis), was inoculated with $N$. gonorrhoeae and $U$. urealyticum.

\section{Materials and methods}

Six adult male Macaca fascicularis weighing between 3 and $8.5 \mathrm{~kg}$ were used. Monkeys were individually caged and fed commercially prepared monkey pellets. Before inoculation and specimen collection the monkeys were sedated with ketamine hydrochloride (Vetalar, Parke-Davis and Co., Detroit, Michigan). Specimens were obtained from the urethra and conjunctiva with calcium alginate urethrogenital swabs, and from the pharynx and anal canal with cotton swabs. Urethral cultures were obtained by serially advancing swabs more proximally. The first swab was used to prepare a Gram stain and it was then placed in transport medium $(0.2 \mathrm{~mol} / 1$ sucrose in $0.02 \mathrm{~mol} / 1$ phosphate buffer, $\mathrm{pH} \mathrm{7.0)}$ for $C$. trachomatis isolation and frozen at $-70^{\circ} \mathrm{C}$. The second swab was used to streak a 
Thayer-Martin culture plate for $N$. gonorrhoeae isolation. The third swab was used to streak an A6 plate (Shepard and Lunceford, 1970) for Mycoplasma hominis and $U$. urealyticum isolation and then it was placed in broth (Holmes et al., 1975) for $U$. urealyticum isolation. A similar procedure was used in the conjunctiva, pharynx, and anal canal except that Gram stain preparations were not made. $C$. trachomatis, $N$. gonorrhoeae, and $U$. urealyticum, were isolated and identified as previously described (Holmes et al., 1975).

Urethral specimens from men with urethral discharge were obtained and cultured for $N$. gonorrhoeae, C. trachomatis, U. urealyticum, and M. hominis (Holmes et al., 1975). Fresh urethral exudate from five men with gonococcal urethritis, diagnosed by Gram stain and later confirmed by culture, was used to inoculate five of the six monkeys. Patients were taken to the primate centre, urethral exudate was obtained on swabs and immediately inoculated into the monkeys. One swab was used to inoculate the urethra and conjunctiva, and a second swab was used to inoculate the pharynx and anal canal of the monkeys. Urethral exudate from one patient was used to inoculate one monkey. Inoculated monkeys were examined, and cultures were taken for $N$. gonorrhoeae, $C$. trachomatis, $U$. urealyticum, and $M$. hominis between three and five, seven, 14, and 28 days after inoculation. The uninoculated monkey was examined and cultures were taken 28 days after the initial study.

Three months after inoculation of urethral exudate from patients, the six monkeys were inoculated with two laboratory strains of $N$. gonorrhoeae that had been passaged approximately 10 times as type 2 colonies. One strain, typical of isolates from men with asymptomatic gonorrhoea, required arginine, hypoxanthine, and uracil for growth, while the other strain, typical of isolates from men with symptomatic gonorrhoea, did not require arginine, hypoxanthine, and uracil for growth (Crawford et al., 1977). Gonococci were streaked on to GC agar base with Kellogg's supplement (Baltimore Biological Laboratories, Baltimore, Maryland) and incubated for 16 hours in a candle extinction jar at $36 \cdot 5^{\circ} \mathrm{C}$. Organisms were suspended in trypticase soy broth at a concentration of $10^{7}$ colony-forming units per $\mathrm{ml}$ as determined by dilution plate counts on GC agar. A catheter was used to inoculate $0.5 \mathrm{ml}$ of broth into the urethra while inoculum-soaked swabs were used to inoculate the conjunctiva, pharynx, and anal canal. Each strain was used to inoculate three monkeys. The six monkeys were examined and cultured two and seven days after inoculation.
Two strains of $U$. urealyticum isolated from the urethra of two men with acute epididymitis associated with nongonococcal urethritis were used to inoculate the monkeys three weeks after inoculation of the $N$. gonorrhoeae cultures. The strains were isolated in broth, and at the onset of colour change denoting a rise in $\mathrm{pH}$, the cultures were frozen at $-70^{\circ} \mathrm{C}$. The cultures were then thawed, passed into new broth, and incubated. To ensure that the inoculum contained only $U$. urealyticum, the organisms were terminally diluted three times. The highest dilution showing colour change in the third passage was subcultured into broth. A 14-hour growth of this subculture was used as the inoculum. It contained $10^{6}$ colour changing units (CCU) of $U$. urealyticum per $\mathrm{ml}$. A catheter was used to inoculate $0.5 \mathrm{ml}$ of inoculum into the urethra, and an inoculum-soaked swab was used to inoculate the conjunctiva. Each strain was used to inoculate three monkeys. Monkeys were examined, and the urethra, conjunctiva, and pharynx were cultured one, two, three, six, and eight weeks after inoculation. Five months after the primary inoculation with the in vitro cultures of $U$. urealyticum, all six monkeys were reinoculated with the same strains of $U$. urealyticum. This inoculum contained $10^{7} \mathrm{CCU}$ per $\mathrm{ml}$ and $0.5 \mathrm{ml}$ was again inoculated into the urethra. Monkeys were examined and the urethra cultured three, seven, $10,14,21$, and 28 days after inoculation.

\section{Results}

\section{BASELINE STUDIES OF MONKEYS}

All specimens obtained between one and five weeks earlier and immediately before inoculation with urethral exudate were negative for $N$. gonorrhoeae, C. trachomatis, and U. urealyticum. Polymorphonuclear leucocytes (PMN) were not seen on Gramstained smears of baseline urethral swabs (Table). All pharyngeal specimens and one anal canal specimen yielded typical mycoplasma colonies which appeared urease-positive on A6 agar. The specimens which yielded these colonies also partially changed the colour of the phenol red $\mathrm{pH}$ indicator in urea broth towards alkaline. Specimens which did not yield these colonies did not alter the $\mathrm{pH}$. However, neither the organisms grown on agar nor those changing the $\mathrm{pH}$ in broth could be passed in either agar or broth. The identity of these organisms was not determined, but the organisms were frequently isolated from the pharnyx, and occasionally from the anal canal throughout the study. 
Table Urethral inoculation of Macaca fascicularis with Ureaplasma urealyticum

\begin{tabular}{|c|c|c|c|c|c|c|c|c|c|c|}
\hline \multirow[b]{3}{*}{$\begin{array}{l}\text { Monkey } \\
\text { no. }\end{array}$} & \multirow{3}{*}{$\begin{array}{l}\text { Pre- } \\
\text { inocula- } \\
\text { tion } \\
\text { PMN* }\end{array}$} & \multicolumn{3}{|c|}{ Urethral exudate inoculated } & \multicolumn{3}{|c|}{ U. urealyticum inoculated } & \multicolumn{3}{|c|}{ U. urealyticum reinoculated } \\
\hline & & \multicolumn{3}{|c|}{ U. urealyticum } & \multicolumn{3}{|c|}{ U. urealyticum } & \multicolumn{3}{|c|}{ U. urealyticum } \\
\hline & & . Inoculum $\dagger$ & $\begin{array}{l}\text { Isolated at } \\
\text { follow-up }+\end{array}$ & $P M N^{*}$ & Inoculum + & $\begin{array}{l}\text { Isolated at } \\
\text { follow-up }\end{array}$ & $P M N^{*}$ & Inoculum $†$ & $\begin{array}{l}\text { Isolated at } \\
\text { follow-up }+\end{array}$ & $P M N^{*}$ \\
\hline 6870 & 0 & $10^{4}$ & No & $0-2$ & $10^{6}$ & No & $3-5$ & $10^{7}$ & 3,7 & $0-1$ \\
\hline 7088 & 0 & $10^{4}$ & No & 0 & $10^{6}$ & $7,14,21,42$ & $3-5$ & $10^{7}$ & No & $0-1$ \\
\hline 70564 & 0 & - & - & 一 & $10^{6}$ & 7 & $0-1$ & $10^{7}$ & 10 & $12-18$ \\
\hline 75231 & 0 & $10^{1}$ & No & $0-2$ & $10^{6}$ & Contaminated & $3-5$ & $10^{7}$ & 7,10 & $3-4$ \\
\hline 75237 & 0 & $10^{1}$ & No & 0 & $10^{6}$ & 7 & $3-4$ & $10^{7}$ & 3,7 & $3-6$ \\
\hline 75242 & 0 & 0 & No & 0 & $10^{6}$ & Contaminated & $0-1$ & $10^{7}$ & 7 & 0 \\
\hline
\end{tabular}

* Range of the number of polymorphonuclear leucocytes per $\times 1000$ oil immersion field on Gram stain of urethral smears

tConcentration of inoculum expressed as the number of colour changing units per ml of first-voided urine in the inoculation of urethral exudate, and per $\mathrm{ml}$ of urea broth for the $U$. urealyticum inoculations

$\ddagger$ Days after inoculation at which $U$. urealyticum was isolated

Inoculation with urethral exudate from men with urethral gonorrhoea

The presence of $N$. gonorrhoeae in patients was confirmed by growth on Thayer-Martin medium of oxidase-positive Gram-negative diplococci which fermented glucose, but not maltose, sucrose, or lactose. In addition, four patients had $10^{1}$ to $10^{4}$ CCU of $U$. urealyticum per $\mathrm{ml}$ of first-voided urine, but it is not known how many $U$. urealyticum organisms were present in the urethral exudate that was inoculated. The results of follow up cultures for $U$. urealyticum are shown in the Table. Two patients also had $C$. trachomatis isolated from the urethral exudate. Monkeys (nos 7088 and 75231) received exudate from these men. However, all follow-up specimens for the inoculated sites in monkeys were negative for $N$. gonorrhoeae, $C$. trachomatis, and $U$. urealyticum. No discharge was observed from any site, and only an occasional PMN per $\times 1000$ oil immersion field was observed on examination of the entire Gram stain.

Inoculation with in vitro cultures of $\mathrm{N}$. gonorrhoeae Specimens from monkeys inoculated with laboratory cultivated gonococci were negative except for one monkey, from which $N$. gonorrhoeae was obtained from the pharynx two days after inoculation. Gram-negative extracellular diplococci were also observed on the urethral smear from this monkey but viable gonococci were not isolated from the urethra. All specimens collected seven days after inoculation gave negative results. No discharge from the urethra or conjunctiva, or inflammation of the conjunctiva, pharynx, or anal canal was observed. Zero to 1 PMN per $\times 1000$ field was observed in urethral smears.

Inoculation with in vitro cultures of $\mathrm{U}$. urealyticum The results are shown in the Table. One week after inoculation, the organism was recovered from the urethra of three monkeys and was not isolated from one. Cultures made from the remaining two monkeys were contaminated with bacteria. Urethral specimens collected two weeks after inoculation were negative except for one monkey in which infection continued for six weeks. $U$. urealyticum was not isolated from the conjunctiva. There was no evidence of a urethral discharge, but four monkeys exhibited 3-5 PMN per $\times 1000$ field on the urethral smear.

Reinoculation with in vitro culture of $\mathrm{U}$. urealyticum Before reinoculation five months after the initial inoculation of $U$. urealyticum, urethral cultures were negative for $U$. urealyticum and no PMN were observed on urethral smears. In five of the six monkeys $U$. urealyticum was reisolated for seven to 10 days after reinoculation (Table), but subsequent cultures were negative. The monkey that had previously shed $U$. urealyticum for six weeks was not reinfected and did not have an increased number of PMN on Gram stain. No urethral discharge was present in the monkeys, but two had 3-6 and one had 12-18 PMN per $\times 1000$ field on the urethral smear.

\section{Discussion}

No infection of the urethia, conjunctiva, pharynx, and anal canal of Macaca fascicularis was produced by inoculating urethral exudate from men with gonorrhoea, or of in vitro cultures of $N$. gonorrhoeae. However, inoculation of in vitro cultures of $U$. urealyticum initially isolated from men with epididymitis, resulted in positive cultures in three of four monkeys with uncontaminated cultures after primary inoculation, and in five of six monkeys after reinoculation. Cultures were only transiently positive in all but one monkey. This does not necessarily indicate that the monkeys were infected, 
but infection is possible because in some of the animals an increased number of PMN was seen on urethral Gram stain concurrent with isolation of $U$. urealyticum. More than 3 PMN per $\times 1000$ field on urethral Gram stain were not seen in the baseline studies, after the inoculations with urethral exudate and $N$. gonorrhoeae, nor after inoculations with $U$. urealyticum at times when cultures for $U$. urealyticum were negative. Except for one monkey, 3 or more PMN per $\times 1000$ field were detected only at times when cultures for $U$. urealyticum were positive. In men attending a sexually transmitted diseases clinic a mean of over 4 PMN per $\times 1000$ field on urethral Gram stain, obtained when the patient had not voided for two hours or longer, correlated with increased numbers of PMN in the sediment of the first-voided urine (Bowie, 1978). The number of PMN seen in the monkeys' urethral exudate is probably significant when it is considered that the monkeys invariably voided during the sedation procedure.

As there were only six monkeys, none was given a control inoculation with urea broth without $U$. urealyticum. It is unlikely that the physical trauma of inoculating the urethra or obtaining repeated urethral cultures produced the increase in numbers of PMN because they did not occur with the inoculations of $N$. gonorrhoeae and the PMN disappeared although further follow-up cultures were obtained. However, the possibility that some component of the urea broth medium elicited the increase in numbers of PMN has not been ruled out.

These studies support clinical studies which implicate $U$. urealyticum as a urethral pathogen in humans. In men with nongonococcal urethritis experiencing their first episode of urethritis, the isolation rate of $U$. urealyticum was significantly higher from $C$. trachomatis negative patients than from $C$. trachomatis positive patients or from men without urethritis who were comparable in sexual history (Bowie et al., 1977). Furthermore, treatment of $C$. trachomatis-negative, $U$. urealyticum-positive urethritis with aminocyclitols resulted in improvement of the urethritis when $U$. urealyticum was eradicated, but not when it persisted (Bowie et al., 1976). Experimental inoculation of the human urethra with $U$. urealyticum has been performed in two men (Taylor-Robinson et al., 1977). Both men developed threads in the urine, and one developed a mucoid discharge.

There are problems with using Macaca fascicularis as a model. The animals invariably micturate during the sedation procedure, and if a urethral discharge is produced it would probably be washed away. Micturition decreases the number of PMN that can be detected on urethral smear and may have interfered with detection of low concentrations of $U$. urealyticum. Because of the small size of the urethra in this species, urethroscopy cannot be performed. Nevertheless, this is the second nonhuman primate in which urethral $U$. urealyticum infection has been produced, and nonhuman primates may possibly serve as an alternative to humans. Such models could be used to explore the relative pathogenicity of various types of $U$. urealyticum, and perhaps to analyse the steps in the pathogenesis of urethritis and the evolution of specific or heterologous immunity.

This study was supported by Public Health Service Grants AI-12192 from the National Institute of Allergy and Infectious Diseases and RR-00166 from the Division of Research Resources, and a Medical Research Council of Canada Fellowship (WRB).

\section{References}

Bowie, W. R. (1978). Comparison of Gram stain and first voided urine sediment in the diagnosis of urethritis. Sexually Transmitted Diseases, in press.

Bowie, W. R., Alexander, E. R., Floyd, J., Holmes, J., Miller, Y., and Holmes, K. K. (1976). Differential response of chlamydial and Ureaplasma-associated urethritis to sulphafurazole (sulfisoxazole) and aminocyclitols. Lancet, 2, 1276-1278.

Bowie, W. R., Wang, S-P., Alexander, E. R., Floyd, J., Forsyth, P. S., Pollock, H. M., Lin, J-S. L., Buchanan, T. M., and Holmes, K. K. (1977). Etiology of nongonococcal urethritis: evidence for Chlamydia trachomatis and U'reaplasma urealyticum. Journal of Clinical Investigation, 59, 735-742.

Crawford, G., Knapp, J. S., Hale, J., and Holmes, K. K., (1977). Asymptomatic gonorrhea in men: caused by gonococci with unique nutritional requirements. Science, 196, 1352-1353.

DiGiacomo, R. F., Gale, J. L., and Holmes, K. K. (1977). Genital inoculation of male baboons with Neisseria gonorrhoeae. Infection and Immunity, 15, 670-671.

DiGiacomo, R. F., Gale, J. L., Wang, S-P., and Kiviat, M. D. (1975). Chlamydial infection of the male baboon urethra. British Journal of Venereal Diseases, 51, 310-313.

Gale, J. L., DiGiacomo, R. F., Kiviat, M. D., Wang, S-P., and Bowie, W. R. (1977). Experimental non-human primate urethral infection with Chlamydia and Ureaplasma (T-mycoplasma). In Nongonococcal Urethritis and Related Infections, pp. 205-213. Edited by D. Hobson and K. K. Holmes. American Society for Microbiology: Washington DC.

Holmes, K. K., Handsfield, H. H., Wang, S-P., Wentworth, B. B., Turck, M., Anderson, J. B., and Alexander, E. R. (1975). Etiology of nongonococcal urethritis. New England Journal of Medicine, 292, 1199-1205.

Lucas, C. T., Chandler, F., Jr, Martin, J. E., Jr, and Schmale, J. D. (1971). Transfer of gonococcal urethritis from man to chimpanzee. An animal model for gonorrhea. Journal of the American Medical Association, 216, 1612-1614.

Shepard, M. C., and Lunceford, C. D. (1970). Differential agar medium for identification of $\mathrm{T}$-mycoplasmas in primary cultures. Bacteriological Proceedings, 70, 83.

Taylor-Robinson, D., Csonka, G. W., and Prentice, M. J. (1977). Human intraurethral inoculation of ureaplasmas. Quarterly Journal of Medicine, 46, 309-326. 